\title{
Total bilirubin level as a biomarker for dampness-heat differentiation in traditional Korean treatment for jaundice
}

\author{
Ki Cheul Sohn', Hyun-Jung Jung ${ }^{2}$, A-Jin Lee ${ }^{3}$, Sang-Gyung Kim, ImHee Shin 5 , \\ Sang Gyu Kwak ${ }^{6}$

\begin{abstract}
${ }^{1}$ Department of Preventive Medicine, Catholic University of Daegu, School of Medicine, Daegu, Korea ${ }^{2}$ Department of Diagnostics, College of Korean Medicine, Daegu Hanny University Comprehensive Integrated Medicine Institute, Daegu, Korea Medicine Institute, Daegu, Korea

${ }^{4}$ Department of Laboratory Medicine, Catholic University of Daegu, School of Medicine, Daegu, Korea

${ }^{6}$ Department of Medical Statistics \& Informatics, School of Medicine, Catholic University of Daegu, Comprehensive Integrated Medicine Institute, Daegu, Korea ${ }_{1,2}$ These two authors contributed equally to this work.
\end{abstract} \\ ${ }^{3}$ Department of Laboratory Medicine, Catholic University of Daegu, School of Medicine, Comprehensive Integrated \\ ${ }^{5}$ Department of Medical Statistics \& Informatics, Catholic University of Daegu, School of Medicine, Daegu, Korea
}

\begin{abstract}
Objectives: Classifying the pattern of jaundice during diagnosis will significantly improve the outcome of common $\mathrm{KM}$ interventions. This study aimed at determining an objective index for accurately diagnosing heat and dampness KM patterns in patients with jaundice.

Methods: We systematically reviewed laboratory findings from case reports published in the scientific literature of Korean medicine. Cases were classified as following either the heat or dampness pattern. Biochemical indices were compared using a Bayesian factor (BF) analysis and standard t-tests.

Results: The laboratory findings of 32 patients were evaluated. The heat pattern was observed in 17 patients and the dampness pattern in 15 . No significant differences were observed between the 2 groups in terms of white blood cell count $(\mathrm{BF}=1.659)$; hemoglobin concentration $(\mathrm{BF}=2.627)$; platelet count $(\mathrm{BF}=1.019)$; or levels of direct bilirubin $(\mathrm{BF}=1.453)$, aspartate aminotransferase $(\mathrm{BF}=1.226)$, alanine aminotransferase $(\mathrm{BF}=1.340)$, alkaline phosphatase $(\mathrm{BF}=2.344)$, or gamma-glutamyl transpeptidase $(\mathrm{BF}=2.782)$. However, total bilirubin levels were significantly higher in the dampness pattern group $(\mathrm{BF}=0.854, P$-value $=0.070)$.

Conclusions: Patients with high total bilirubin levels may predominantly follow the dampness pattern, while those with low levels may predominantly follow the heat pattern. These results are expected to be useful for the development of timely and efficient KM treatments as well as new integrative therapeutic approaches for jaundice. However, further studies are essential to fully validate the utility of total bilirubin as a biomarker for differentiating between heat and dampness patterns.
\end{abstract}

\section{Key Words : jaundice; differential diagnosis; bilirubin; integrative medicine; traditional Korean medicine}

\section{Introduction}

Integrative medicine is generally defined as a blend of conventional and complementary and alternative medicine (CAM), aimed at providing the best care for the patient as a whole, by applying the most appropriate options from each modality ${ }^{1}$. Over the past 2 decades, there has been a documented

- Received : 31 October 2013

- Revised : 13 December 2013

- Accepted : 18 December 2013

- Correspondence author: A-Jin Lee, M.D.

Department of Laboratory Medicine, Catholic University of Daegu, School of Medicine, Comprehensive Integrated Medicine Institute, Daegu, Korea 3056-6, Daemyeong 4-dong, Nam-gu, Daegu 705-718, Korea Tel : +82-53-650-4140, Fax : +82-53-653-8672, Email : ajlee388@gmail.com 
increase in the number of clinical centers providing integrative medicine worldwide, including in the United States, Europe, and Korea ${ }^{2,3)}$. Traditional medical systems such as traditional Chinese medicine (TCM), Ayurveda, and traditional Korean medicine (KM) are considered to be components of CAM and have played key roles in the development of integrative medicine. Integrative medicine can help clinicians and practitioners address the patient's whole being and experience, potentially meeting the patient's total needs more completely than western medicine (WM) or TCM alone could ${ }^{4}$.

The number of hospitals administering integrative medicine and the number of patients seeking integrative medical service are increasing every year. In addition, recent studies have shown that TCM or $\mathrm{KM}$, in the form of acupuncture and herbal medicine, have positive effects for some symptoms and diseases such as cancer-related pain, allergic rhinitis, and degenerative arthritis ${ }^{5-7)}$. However, documentation and successful integration of TCM and $\mathrm{WM}$ in clinical practice is lacking ${ }^{8)}$.

There are several differences between TCM and conventional medicine. In TCM, a symptom or sign can only be understood in terms of how it relates to the integrity of the whole patient, with particular consideration for environmental interactions ${ }^{5)}$. In $\mathrm{WM}$, diagnoses are based on scientific knowledge and treatments have been proven effective through scientific research. Generally, TCM is relatively qualitative, while $\mathrm{WM}$ is relatively quantitative ${ }^{8}$. However, the application of TCM pattern differentiation has been demonstrated to be effective in clinical practice ${ }^{9)}$ KM is partly based on TCM and has many common features. However, KM focuses on the specific characteristics of the patient rather than individual symptoms ${ }^{10}$. $\mathrm{KM}$ follows the pattern differentiation of symptom and approach of TCM (bianzheng shizhi), but focuses on observed symptoms and their relation to the patient's physical characteristics $^{11)}$. Therefore, KM sometimes uses different treatments for the same disease, the choice of which depends on the patient's physical characteristics. KM has been found to be highly effective and is widely used in chronic diseases and disorders that do not respond to WM. Therefore, integrative approaches are expected to be very successful and efficient.

Jaundice is one of the main symptoms of hepatobiliary disease. Jaundice can be classified in both WM and KM, but each has a different approach to classification. Commonly, jaundice is defined as yellowing of the skin. From the WM perspective, jaundice is classically defined as a serum bilirubin level $>2.53 \mathrm{mg} / \mathrm{dL}$ along with a clinical finding of yellowish skin and sclera caused by an excessive deposition of bilirubin secondary to hyperbilirubinemia. Skin discoloration is typically detected clinically once the serum bilirubin level has increased above 3 $\mathrm{mg} / \mathrm{dL}$. Bilirubin metabolism takes place in 3 phases: pre-hepatic, intra-hepatic, and post-hepatic. Dysfunction in any of these phases can lead to jaundice. Jaundice is a consequence of many different clinical conditions, including hepatobiliary, cardiac, hematologic, and pancreatic disorders. From the perspective of KM, there are many different methods of classifying jaundice, which are based on various theories. Heat and dampness are the main etiological factors of jaundice. Patients presenting with the heat pattern tend to have symptoms such as anorexia, anxiety, chest pain, chest discomfort, dry mouth, thirst, reddish urine, and constipation. Patients presenting with the dampness pattern tend to have symptoms such as abdominal swelling, nausea, vomiting, fatigue, yellowish urine, brownish urine, and sticky stool.

A combination of conventional and complementary and alternative therapy could be useful to cure jaundice. Several studies have concluded that KM is of great utility in the cure of jaundice ${ }^{12-14)}$. A variety of studies have focused on the therapeutic effects of $\mathrm{CAM}^{15-18)}$. Therefore, it is expected that integrating 
$\mathrm{KM}$ and WM could significantly improve the efficacy of therapeutic interventions for jaundice. However, to effect such an integration of $\mathrm{KM}$ and WM therapies, it is first necessary to integrate their different diagnostic classifications of jaundice.

The objective of the present study was to classify jaundice case reports according to the KM heat vs. dampness pattern and to compare this classification with WM laboratory test results. Signs, symptoms, and laboratory test results were systemically reviewed from case reports published in the Korean scientific literature. Efforts were made to determine if any diagnostic associations exist between the WM and $\mathrm{KM}$ perspectives and whether such associations could be used to develop integrative therapy.

\section{Methods}

\section{Literature Sources and Search Strategy}

Figure 1 presents an overview of our literature search strategy, including the number of studies included and excluded at each stage in the process. We searched the main Korean electronic databases, including the Oriental Medicine Advanced Searching Integrated System (OASIS), the National Assembly Library, the Korean studies Information Service System (KISS), the National Discovery for Science Leader (NDSL), and the DBpia Korean e-journal portal. Our search terms included ("Jaundice") AND ("Case study" OR "Case report" OR "Case"). For some databases (National Assembly Library, OASIS), we only used the search term "Jaundice." Institutional review board and signed consent from patients were not required because all information used in our analyses had already been published.

\section{Inclusion Criteria}

We included case reports and clinical experiences published in Korean journals if patients' symptoms and blood test results were both described and if the patients were diagnosed with or complained of jaundice. To be included, articles also needed to contain enough information to allow us to distinguish between the dampness and heat patterns (28 articles) (Fig. 1).

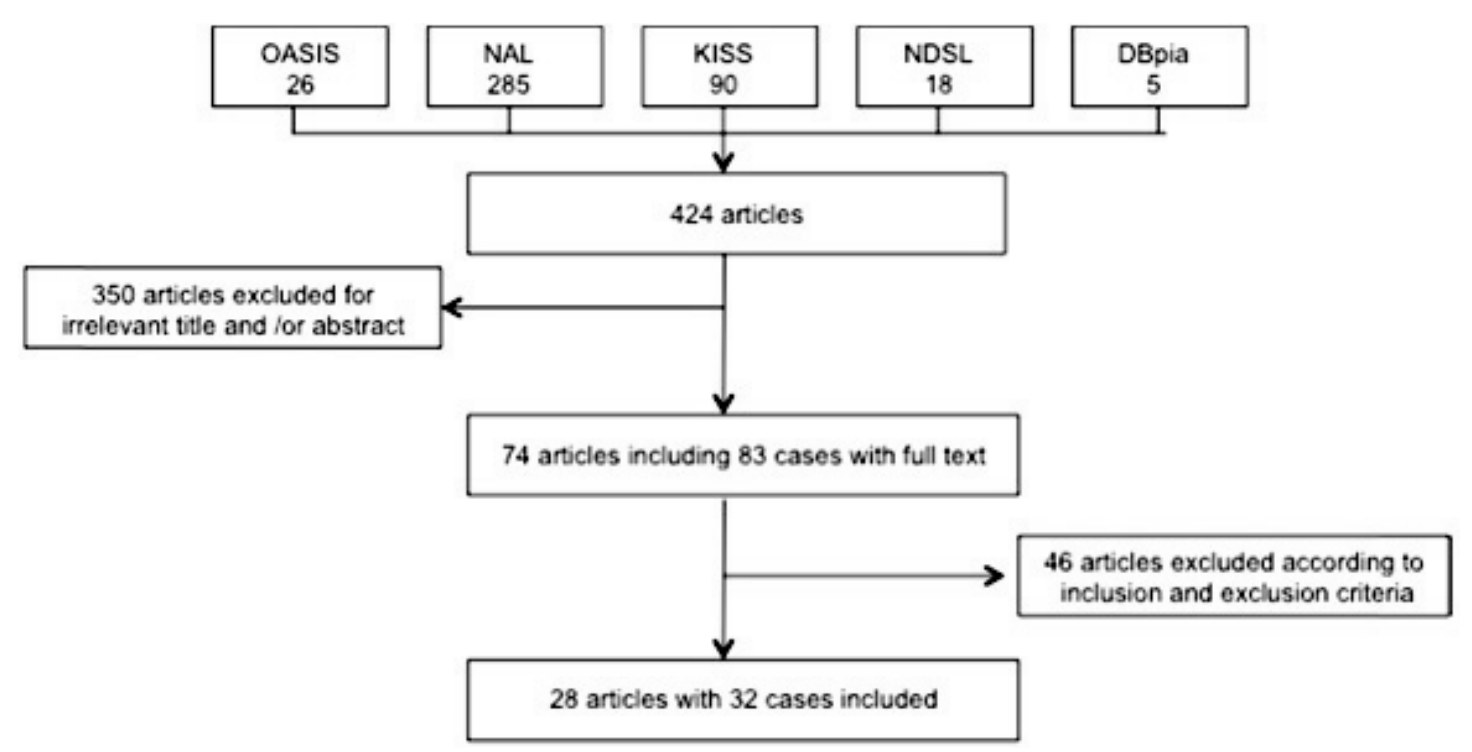

Fig. 1. Flowchart of the literature search and study selection 


\section{Exclusion Criteria}

All case series, reports of in vitro cell cultures, and animal studies were excluded. For each article that was not excluded based on an irrelevant title and/or abstract (350 articles), the full article text was evaluated (74 articles). We also excluded case reports that did not list 5 or more symptoms (to distinguish between the dampness and heat patterns), and all case reports for which the authors' classification assignments (heat vs. dampness) did not coincide (46 articles).

After applying the inclusion and exclusion criteria, 28 articles involving 32 patients remained and were analyzed in this study (Fig. 1).

\section{Distinguishing between dampness and heat pattern}

According to the Dongeui Bogam ${ }^{19)}$, heat and dampness are the two main etiological factors of jaundice, so jaundice can be subdivided into the heat and dampness patterns. The heat pattern is characterized by heat intolerance, red complexion, anxiety, constipation, red urine, thirst, mouth dryness, hot limbs, thickly coated tongue, dense or thick discharge. In contrast, the dampness pattern is characterized by chest discomfort, nausea, vomiting, languidness, vaginal discharge, edema, or diarrhea. Two doctors of KM reviewed each case that met our inclusion criteria. We classified a case as following the dampness or heat pattern only if the judgment of both doctors agreed.

\section{Laboratory Test Results}

Laboratory test results were obtained from published case records. We compared these laboratory data according to the patient group. Complete blood cell counts included white blood cell count (WBC), hemoglobin (Hb) concentration, hematocrit (Hct) level, and platelet count (PLT). Liver function tests included those measuring the aspartate aminotransferase (AST), alanine aminotransferase (ALT), alkaline phosphatase (ALP), gamma glutamyl transpeptidase (GGT), direct bilirubin, and total bilirubin levels. Protein, albumin, blood urea nitrogen (BUN), and creatinine levels were also compared.

\section{Statistical Methods}

The two-sample $t$-test and Bayes factor (BF) were used to compare groups in order to test for differences between mean variable values in the heat and dampness groups (our null hypothesis was no difference between heat and dampness groups). The two-sample $t$-test and $\mathrm{BF}$ are frequentist and Bayesian approaches, respectively. For each variable in our analysis, we also compared the frequentist and Bayesian test statistics with each other.

Bayesian methodology is an innovative way to differentiate KM patterns for jaundice. Broadly, the $\mathrm{BF}$ is a ratio of the odds of 2 models (null and alternative), expressed in decimal form. BF can also be thought of as the odds that result from dividing the likelihood of the null hypothesis by the likelihood of the alternative hypothesis. The BF can be regarded as the result of a simple fraction or division, in which the probability of null hypothesis is divided by the probability of alternative hypothesis. Its interpretation is straightforward: a $\mathrm{BF}$ of 1.0 represents equal odds for either hypothesis, a $\mathrm{BF}$ greater than 1.0 suggests evidence for the null hypothesis, and a BF less than 1.0 suggests evidence for the alternative hypothesis.

As for traditional effect size estimates, the interpretation of $\mathrm{BF}$ involves some flexible categories $^{20)}$. For instance, a BF between approximately 1.0 and 3.0 (or 0.3 and 1.0 ) represents weak evidence for the null hypothesis (or alternative hypothesis); a BF between approximately 3.0 and 10.0 (or 0.1 and 0.3 ) represents substantial evidence 
for the null hypothesis (or alternative hypothesis); a BF between approximately 10.0 and 30.0 (or 0.03 and 0.1) represents strong evidence for the null hypothesis (or alternative hypothesis); and a BF between approximately 30.0 and 100.0 (or 0.03 and 0.01 ) represents very strong evidence for null hypothesis (or alternative hypothesis).

In this study, the null hypothesis was 0 difference between the mean values of a variable for the heat and dampness patterns $\left(\mathrm{H}_{0}: \mu_{\mathrm{A}}-\mu_{\mathrm{B}}=0\right.$, where $\mu_{\mathrm{A}}$ is the mean value of the variable for the heat pattern group, and $\mu_{\mathrm{B}}$ is the mean value for the dampness pattern group). The alternative hypothesis was a non-zero difference between the mean value of a variable in the heat and dampness pattern groups $\left(\mathrm{H}_{1}: \mu_{\mathrm{A}}-\mu_{\mathrm{B}} \neq 0\right)$. Therefore, a BF greater than 1.0 does not suggest the existence of difference between the means of the 2 groups. On the other hand, a BF less than 1.0 suggests that such a difference exists.

We used open-source R software (version 2.15.2), which is the result of a collaborative effort with contributions from all over the world, and the "LearnBayes" package ${ }^{21)}$ for statistical computations.

\section{Results}

\section{Bibliometric Information}

We screened a total of 424 articles that were obtained by searching the 5 electronic databases. Of these articles, 350 were excluded due to irrelevant title and/or abstract. For each of the remaining 74 articles (containing 83 cases), the full texts were evaluated. Forty-six articles were excluded because they did not contain enough information on symptoms to distinguish classify according to the heat and dampness patterns.

\section{Patient Characteristics}

In total, 28 articles involving 32 patients were

Table 1. Comparison of the Results by $\mathrm{t}$-test $(P$-value) and Bayes Factor for Jaundice-related Variables

\begin{tabular}{|c|c|c|c|c|c|c|c|}
\hline \multirow{3}{*}{ Variable } & \multicolumn{4}{|c|}{ Group } & \multirow{3}{*}{$\mathrm{t}(P$-value $)$} & \multicolumn{2}{|c|}{ Bayes Factor (BF) } \\
\hline & \multicolumn{2}{|c|}{ Heat pattern $^{1}$} & \multicolumn{2}{|c|}{ Dampness pattern $^{2}$} & & \multicolumn{2}{|l|}{ Prior } \\
\hline & $\mathrm{N}$ & Mean (SD) & $\mathrm{N}$ & Mean (SD) & & Cauchy dist. & Normal dist. \\
\hline $\mathrm{BT}\left({ }^{\circ} \mathrm{C}\right)$ & 13 & $37.69(1.16)$ & 10 & $36.64(0.66)$ & $2.735(0.013)^{*}$ & $0.225^{*}(1>2)$ & $0.160^{*}(1>2)$ \\
\hline $\mathrm{HR}$ (/min) & 9 & 84.89 (19.93) & 9 & $84.22(12.14)$ & $0.086(0.933)$ & 3.100 & 2.338 \\
\hline $\mathrm{WBC}\left(10^{3} / \mu \mathrm{L}\right)$ & 13 & $12.0(10.1)$ & 9 & $6.1(1.7)$ & $-0.594(0.579)$ & 1.659 & 1.242 \\
\hline $\mathrm{Hb}(\mathrm{g} / \mathrm{dL})$ & 12 & $11.1(2.9)$ & 10 & $24.5(37.8)$ & $-1.270(0.219)$ & 2.627 & 1.960 \\
\hline Het (\%) & 7 & $33.7(8.0)$ & 7 & $37.4(6.2)$ & $-0.931(0.370)$ & 1.973 & 1.448 \\
\hline $\operatorname{PLT}\left(10^{3} / \mu \mathrm{L}\right)$ & 12 & $160(96)$ & 8 & $233(83)$ & $-1.753(0.098)$ & 1.019 & $0.730^{*}(1<2)$ \\
\hline AST (IU/L) & 17 & $675(826)$ & 14 & $267(533)$ & $1.663(0.108)$ & 1.226 & $0.885^{*}(1>2)$ \\
\hline $\operatorname{ALT}(\mathrm{IU} / \mathrm{L})$ & 17 & $675(992)$ & 14 & 225 (394) & $1.595(0.122)$ & 1.340 & $0.970^{*}(1>2)$ \\
\hline ALP (IU/L) & 14 & $608(507)$ & 9 & $359(454)$ & $1.196(0.245)$ & 2.344 & 1.737 \\
\hline GGT (IU/L) & 10 & $432(506)$ & 5 & $437(509)$ & $-0.021(0.983)$ & 2.782 & 2.081 \\
\hline Total bilirubin (mg/dL) & 16 & $7.00(6.64)$ & 14 & $13.86(11.85)$ & $-1.917(0.070)$ & $0.854^{*}(1<2)$ & $0.608^{*}(1<2)$ \\
\hline Direct bilirubin (mg/dL) & 11 & $4.94(5.52)$ & 8 & $9.08(7.29)$ & $-1.413(0.176)$ & 1.453 & 1.052 \\
\hline Protein $(\mathrm{g} / \mathrm{dL})$ & 12 & $6.6(0.9)$ & 7 & $6.3(0.6)$ & $0.855(0.405)$ & 2.313 & 1.711 \\
\hline Albumin (g/dL) & 13 & $3.4(0.6)$ & 7 & $3.2(0.4)$ & $0.994(0.333)$ & 2.115 & 1.557 \\
\hline BUN (mg/dL) & 5 & $17.9(10.5)$ & 4 & $33.4(38.9)$ & $-0.865(0.416)$ & 1.854 & 1.363 \\
\hline Creatinine (mg/dL) & 5 & $1.0(0.4)$ & 4 & $3.9(6.1)$ & $-0.971(0.403)$ & 1.737 & 1.276 \\
\hline
\end{tabular}

*: Statistically significant with $P<0.05$ or $\mathrm{BF}<1$

BT, body temperature; HR, heart rate; WBC, white blood cell count; Hb, hemogloblin; Hct, hematocrit; PLT, platelet count; AST, aspartate aminotransferase; ALT, alanine aminotransferase; ALP, alkaline phosphatase; GGT, gamma glutamyl transpeptidase; BUN, blood urea nitrogen. 


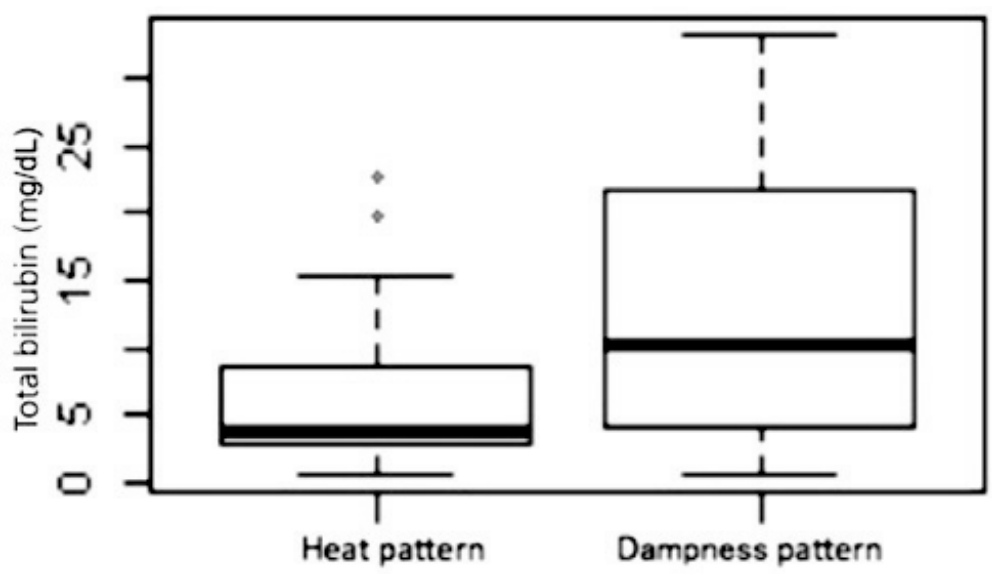

Fig. 2. Comparisons of the level of total bilirubin between the heat and dampness pattern groups. Patients in the dampness pattern group generally had higher total bilirubin levels.

included in the present study's results. The mean age of patients was 46 years (range 3-77). Nineteen patients were female and 13 were male. Only 7 patients were treated by KM. Seventeen patients were categorized as belonging to the heat pattern group (53\%), and 15 patients were categorized as belonging to the dampness pattern group 15 (47\%). On an average, each article provided information on about 7 symptoms.

\section{Laboratory data}

1) Vital signs

Key patient vital signs are presented in Table 1 . Body temperature (BT) was found to be significantly higher in the patients showing heat pattern $(\mathrm{BF}=$ $0.225, P$-value $=0.013)$. Heart rate $(\mathrm{HR}) \mathrm{did}$ not differ significantly between the 2 groups $(\mathrm{BF}=$ 3.100, $P$-value $=0.933$ ).

2) Complete blood cell counts

Key laboratory data are summarized in Table 1 . A statistically significant difference between the 2 groups was not observed for any of these variables, either by the BF or the $t$-test analysis.

3) Liver function tests
Key laboratory test data are summarized in Table 1. For several variables, i.e., AST, ALT, ALP, GGT, and direct bilirubin, there was weak evidence supporting the null hypothesis (zero difference between the means of the heat and dampness pattern groups). However, for the total bilirubin analysis the $\mathrm{BF}$ was 0.854. Therefore, there is evidence of a non-zero difference in mean total bilirubin levels between the heat and dampness pattern groups. Indeed, as presented in Fig. 2, levels of total bilirubin were significantly higher in the dampness pattern group $(13.86 \pm 11.85 \mathrm{mg} / \mathrm{dL})$ than in the heat pattern group $(7.00 \pm 6.64 \mathrm{mg} / \mathrm{dL})$.

\section{Discussion}

Pattern differentiation is the key theory underlying $\mathrm{KM}$ and provides the most important diagnostic principle for $\mathrm{KM}$ therapy. While $\mathrm{KM}$ pattern differentiation generally focuses on patient characteristics, pattern differentiation in conventional medicine is based on signs and biomedical test results such as laboratory test data. Therefore, patients suffering from the same disease (as diagnosed through conventional medicine) can be classified into different KM patterns. For example, 
jaundice a single WM diseasemay be classified as belonging to the heat or dampness patterns. Researchers have made many attempts to combine $\mathrm{KM}$ and $\mathrm{WM}$ for the treatment of stroke, musculoskeletal disorders, and conditions that result in pain ${ }^{21-26)}$. By integrating conventional medicine with $\mathrm{KM}$ pattern differentiation, clinicians could practice combined health care, helping them meet demands for high-quality medical service. Such demands have been increasing recently, especially for integrative medicine. Therefore, integration of $\mathrm{KM}$ and $\mathrm{WM}$ is essential for future clinical practice. In the present study, we explored whether the laboratory test results differ between heat pattern and dampness pattern jaundice patients.

According to a frequentist $t$-test with an alpha of 0.05 , there was no significant difference in the mean total bilirubin levels between these 2 groups ( $P$-value $=0.070)$. However, according to a Bayesian approach, there was evidence of a difference in the mean levels between the 2 groups $(\mathrm{BF}=0.854)$. Therefore, patients who had dampness pattern jaundice tended to have higher levels of total bilirubin than those with heat pattern jaundice. In comparison, for direct bilirubin, there was no significant difference between the 2 groups according to either method. Accordingly, patients with the dampness pattern may have higher levels of indirect bilirubin. These results are in contrast with the findings of $\mathrm{Wu}$ et $a .^{27)}$ showing that the ratio of direct bilirubin to indirect bilirubin equals or exceeds 1 in patients for whom dampness is dominant.

It has been demonstrated that the syndrome of dampness is caused by an imbalance of water metabolism, either locally or in the whole body ${ }^{27)}$. The pathophysiological basis of dampness-heat syndrome is pathological changes of tongue, which may be one of the important indices by which the dominance of dampness or heat can be judged ${ }^{28)}$. Additionally, we have shown that the level of total bilirubin could also be an index that differentiates between the dampness and heat patterns. For patients with higher levels of total bilirubin, the dampness pattern is more probable.

In WM, the bilirubin level is a biomarker with several uses. Levels of bilirubin can be an important laboratory parameter for differentiating between benign and malignant underlying causes among patients with obstructive jaundice ${ }^{29}$. Higher bilirubin levels may be associated with poor survival rates in patients with pancreatic cancer ${ }^{30}$. In addition, total bilirubin can be used to monitor the treatment of severe jaundice ${ }^{31)}$. When we subdivided jaundice into the heat pattern and the dampness pattern, our criteria were entirely based on symptoms. Consequently, there is no correlation between severity of jaundice and the patterns of heat or dampness.

Indirect bilirubin is the difference between total and direct bilirubin and, accordingly, is a measure of the unconjugated fraction of bilirubin. In WM, indirect hyperbilirubinemia is associated with hemolysis, drug toxicity, and genetic syndromes, such as Gilbert syndrome and hematoma ${ }^{32)}$.

Patients with heat pattern $\left(37.69 \pm 1.16^{\circ} \mathrm{C}\right)$ tended to show higher $\mathrm{BT}$ than those with dampness pattern $\left(36.64 \pm 0.66^{\circ} \mathrm{C}\right)$. BT tended to be higher among patients with heat pattern because heat pattern characterized by heat intolerance. In addition to total bilirubin, our study supports the conclusion that BT can be used for objective assessment of pattern differentiation $(\mathrm{BF}=0.225 ; P$-value $=0.013)$.

Except for total bilirubin, no statistically significant differences were observed between liver function variables for heat pattern and dampness pattern. In KM, dampness-heat syndrome can be caused by a combination of dampness and heat. When dampness-heat accumulates in the liver and gallbladder, jaundice can be a resulting symptom ${ }^{33}$. In WM, liver function tests are often used as a screening tool. Biochemical markers such as AST, ALT, ALP, GGT, protein, and albumin levels can be used to detect the functional status of the liver. 
Despite this, among potential liver biomarkers, only total bilirubin levels were associated with a statistically significant difference between the heat and dampness pattern groups.

Complete blood cell count test results such as WBC count, $\mathrm{Hb}$ concentration, Hct level, and PLT count revealed no differences between the 2 groups. From the WM perspective, these tests reflect hematopoietic function and general conditions such as acute inflammation. BUN and creatinine levels are indicators of kidney function. Consequently, the lack of association between these markers and the 2 groups was expected.

The limitations of this study include that pattern differentiation by symptoms could be subjective, and that pattern typing might differ between traditional medicine practitioners. To address this, two $\mathrm{KM}$ doctors reviewed each case; the case was classified the dampness or heat pattern only if the judgment of both doctors agreed. Also, we only observed published case reports instead of patients' cases, so the authors didn't have enough information to decide pattern. This study also had limitations stemming from its relatively small sample size of reviewed cases. Finally, there is one additional limitation that follows from our choice of Bayesian methods for statistical analysis: such methods require complex computations derived according to complicated formulas. However, it is easy to calculate the value of BF nowadays, thanks to computational software.

From the WM perspective, most cases of jaundice can be treated with the same regimens to relieve symptoms. However, from the KM perspective, different herbal preparations should be prescribed according to different patterns. In this study, we discovered a kind of association between pattern differentiation and laboratory test results. We show that the level of total bilirubin could be a referable index that differentiates between the dampness and heat patterns. Therefore, we suggest that herbal preparations compatible with dampness pattern could be prescribed to patients who have relatively high levels of total bilirubin. However, further study is necessary to verify these results.

\section{Conclusions}

This preliminary study suggests that total bilirubin levels are a potential biomarker for jaundice syndrome types. At high total bilirubin levels, the dampness pattern may predominate, whereas at low total bilirubin levels, heat pattern may predominate. Differentiating between these patterns will help to classify jaundice patients into subgroups that can receive different herbal preparations, which may help to eliminate jaundice.

\section{Abbreviations}

KM: Korean medicine

BF: Bayesian Factor

CAM: complementary and alternative medicine

TCM: traditional Chinese medicine

WM: Western medicine

WBC: white blood cell count

$\mathrm{Hb}$ : hemoglobin

Hct: hematocrit

PLT: platelet count

AST: aspartate aminotransferase

ALT: alanine aminotransferase

ALP: alkaline phosphatase

GGT: gamma glutamyl transpeptidase.

BUN: blood urea nitrogen

BT: body temperature

HR: heart rate

\section{Acknowledgments}

This study was supported by a grant from Ministry of Health \& Welfare, Republic of Korea (Project No: 2011-0-090-091-3000-3033-320) 


\section{References}

1. Royal Australian College of General Practitioners. RACGP curriculum for Australian general practice. Curriculum statement: integrative medicine. 2007; Available at: URL:http://www.racgp.org.au/scriptcontent/curr iculum/pdf/integrativemedicine.pdf. Accessed March 24, 2009.

2. Horrigan B, Lewis S, Abrams DI, Pechura C. Integrative medicine in Americahow integrative medicine is being practiced in clinical centers across the United States. Global Advances in Health and Medicine 1.3. 2012:18-94.

3. Lee SR. Medical services system cooperation between Western and Oriental medicine. The Journal of Daegu Health College. 2009;29: $1-28$.

4. Rosenthal DS, Dean-Clower E. Integrative medicine in hematology/oncology: benefits, ethical considerations, and controversies. Hematology Am Soc Hematol Educ Program. 2005:491-497.

5. Choi TY, Lee MS, Kim TH, Zaslawski C, Ernst E. Acupuncture for the treatment of cancer pain: a systematic review of randomised clinical trials. Support Care Cancer. 2012;20: 147-58.

6. Choi SM, Park JE, Li SS, Jung H, Zi M, Kim $\mathrm{TH}$, et al. A multicenter, randomized, controlled trial testing the effects of acupuncture on allergic rhinitis. Allergy. 2013;68:365-374.

7. Saleki M, Ahadi T, Razi M, Raeisi GR, Forough B, Ali MK. Comparison of the effects of acupuncture and isometric exercises on symptom of knee osteoarthritis. Int $\mathrm{J}$ Prev Med. 2013;4:S73-S77.

8. Giordano J, Garcia MK, Strickland G. Integrating Chinese traditional medicine into a US public health paradigm. J Altern Complement Med. 2004;10:706-710.
9. Lu AP, Jia HW, Xiao C, Lu QP. Theory of traditional Chinese medicine and therapeutic method of diseases. World J Gastroenterol. 2004; 10:1854-1856.

10. Cha WS, Oh JH, Park HJ, Ahn SW, Hong SY, Kim NI. Historical difference between traditional Korean medicine and traditional Chinese medicine. Neurol Res. 2007;29 (Supplement 1):S5-S9.

11. Lee SG. A comparative study on the differenced of mental illness between Korea and China in Oriental medicine. J of Oriental Neuropsychiatry. 2005;16:193-200.

12. Kim DW. Disease pattern identification in Oriental medicine and herb medicine treatment for acute hepatitis A with jaundice: Case series of 3 patients. Korean J Oriental Physiology \& Pathology. 2010;24:696-701.

13. Kim HY, Jang SY, Yoon KM, Im EY, Yoo $\mathrm{HJ}$, Choi GH, et al. A clinical report on 1 case of jaundice caused by the alcoholic liver disease. The Journal of East-West Medicine. 2009;34:37-44.

14. Lee KS, Choi KJ, Lee TG, Koh BH, Song IB, Lee SK. A case study of soeumin jaundice patient diagnosed as drug-induced hepatitis. J of Sasan Constitut Med. 2005;17:99-106.

15. Ferrucci LM, Bell BP, Dhotre KB, Manos MM, Terrault NA, Zaman A, et al. Complementary and alternative medicine use in chronic liver disease patients. J Clin Gastroenterol. 2010;44:e40-e45.

16. Mullin GE. The use of complementary and alternative medicine for liver disease: Part I. Nutr Clin Pract. 2012;28:138-139.

17. MacPherson H, Sinclair-Lian N, Thomas K. Patients seeking care from acupuncture practitioners in the UK: A national survey. Complement Ther Med. 2006;14:20-30.

18. Lu C, Zha Q, Chang A, He Y, Lu A. Pattern differentiation in traditional Chinese medicine 
can help define specific indications for biomedical therapy in the treatment of rheumatoid arthritis. J Altern Complement Med. 2009;15:1021-5.

19. Heo J. Dongguei bogam. 1st ed. Seoul:Bubinbooks. 1999:1345-53.

20. Jeffreys H. Theory of Probability. USA:Oxford University Press. 1961:432-3.

21. Albert J. Bayesian Computation With R. USA:Springer Science+Business Media. 2011: 19-20.

22. Lee MS, Shin B-C, Choi T-Y, Kim J-I. Randomized clinical trials on Eastern-Western integrative medicine for health care in Korean literature: A systematic review. Chin J Integr Med. 2011;17:4851.

23. Nam DW, Choi YS, Kim HB, Kim JI, Lim S, Kim KS, et al. Randomized controlled trial of east-west collaborate medical treatment on female chronic shoulder pain patients. J Korean Acupunct Moxib Soc. 2007;24:113-122.

24. Jung IT, Lee SH, Kim SY, Cha WH, Kim KS, Lee DI, et al. A clinical study of east-west pain treatment on chronic headache patients. J Korean Acupunct Moxib Soc 2005;22:93-104.

25. Kwon NH, Shin YJ, Kim CY, Koh PS, Yi WI, Joh BJ, et al. Comparative clinical study between Oriental medicine and Oriental-Western medical treatment on Bell's palsy. J Korean Acupunct Moxib Soc. 2008;25:19-28.

26. Kim Y-S, Wang J, Mann D, Gaylord S, Lee H-J, Lee M. Korean Oriental medicine in stroke care. Complement Health Pract Review. 2005;10:105-17.

27. Wu XZ, Chen D. Study on objectivation of syndrome typing of jaundice. Zhongguo Zhong Xi Yi Jie He Za Zhi (Chin). 2005;25:773-6.

28. Zhuo DT, Lu JY, Huang LP. Effect of modified huopoxialing decoction in treating pi-wei dampness-heat syndrome and its impact on the tongue epithelial cell apoptosis. Zhongguo Zhong Xi Yi Jie He Za Zhi (Chin). 2011;31:168-71.

29. Garcea G, Ngu W, Neal CP, Dennison AR, Berry DP. Bilirubin levels predict malignancy in patients with obstructive jaundice. HPB (Oxford). 2011;13:426-30.

30. Nakata B, Amano R, Kimura K, Hirakawa K. Comparison of prognosis between patients of pancreatic head cancer with andwithout obstructive jaundice at diagnosis. Int J Surg 2013;doi: 10.1016/j.ijsu.2013.02.023.

31. Xiang HL, Han T, Du Z, Li T, Li J, Lü HM, et al. Analysis of prognostic factors of severe jaundice patients using HB-H-6 resin plasma perfusion. Zhongguo Wei Zhong Bing JiJiu Yi Xue (Chin). 2012;24(6):3303.

32. Thapa BR, Walia A. Liver function tests and their interpretation. Indian J Pediatr. 2007;74: 66371.

33. World Health Organization. WHO international standard terminologies on traditional medicine in the Western Pacific region. WHO Library Cataloguing in Publication Data. 2007. 